\title{
Malignant Adrenal Cortex Neoplasm
}

National Cancer Institute

\section{Source}

National Cancer Institute. Malignant Adrenal Cortex Neoplasm. NCI Thesaurus. Code C9327.

A primary or metastatic malignant neoplasm affecting the adrenal cortex. 